\title{
Visualizing and Quantifying Spinodal Decomposition in a Duplex Stainless Steel
}

\author{
Sarah Mburu ${ }^{1}$, R. Prakash Kolli ${ }^{1}$, Daniel E. Perea ${ }^{2}$, Jia Liu ${ }^{2}$, Samuel C. Schwarm ${ }^{1}$ and Sreeramamurthy
} Ankem $^{1}$

1. Department of Materials Science and Engineering, University of Maryland, College Park, USA.

2. Environmental and Molecular Sciences Laboratory, Pacific Northwest National Laboratory, Richland, USA.

Spinodal decomposition is the phase decomposition of a supersaturated alloy that when quenched is within the miscibility gap. The solid solution is unstable due to negative curvature of the free energy curve, i.e. $\partial^{2} F / \partial C^{2}<0$, and there is no thermodynamic barrier for continuous separation to a more stable phase and the only limitation is kinetic (diffusional) [1]. Small compositional fluctuations form globally throughout the supersaturated solid solution that become larger spatially and in composition leading to the formation of enriched domains. In the case of cast duplex stainless steels (CDSS) such as CF-3 and CF-8, the $\delta$-ferrite phase decomposes into Fe-rich $\alpha$-domain and a Cr-rich $\alpha$-domain [2]. From the context of a structure-property relationship, the mechanism is important since it may cause mechanical property changes such as embrittlement in CDSS during service.

Various characterization techniques have been employed to verify and understand spinodal decomposition. The two important parameters that define compositional fluctuations of an element $i$ are wavelength, $\lambda_{i}$, which defines the spatial extent, and amplitude, $A_{i}$, which defines the compositional extent. Of the characterization techniques available to measure these parameters, an important technique is atom probe tomography (APT). The technique has high spatial resolution and high spectral resolution that permits quantifying the nanoscale compositional fluctuations [3]. Several analysis methods have been developed but there exist challenges in quantifying both parameters. For example, the auto correlation function (ACF) is often used to measure the quantity $\lambda$, but the results can be influenced by step size that can lead to variability of the wavelength [4]. Similarly, the Langer-Bar-onMiller (LBM) method is used to measure the quantity $A$ but the choice of block size can lead to significant changes in the frequency distribution diagrams and further it does not provide accurate values during late stages of spinodal decomposition and is also dependent on the symmetry of the frequency distribution diagrams [4]. Additionally, the two methods are often utilized independently of each another.

We present a method to visualize and measure the quantities $\lambda$ and $A$, from APT reconstructions of CF- 8 CDSS. The method captures the early stages of spinodal decomposition and illustrates the increase of the quantities $\lambda$ and $A$ for chromium $(\mathrm{Cr})$ during isothermal aging. We also illustrate that the method is sensitive to capture differences between specimens exposed to various aging temperatures. The unaged CF-8 steel exhibits incipient or early stages of spinodal decomposition that occurs during solution treatment as illustrated by the alternating Fe-rich $\alpha$-domains (blue) and $\mathrm{Cr}$-rich $\alpha$-domains (magenta) in an example region of interest (ROI), Fig. 1(a). The size of the fluctuations increase in an example region of interest (ROI) during isothermal aging at $400{ }^{\circ} \mathrm{C}$ to $4300 \mathrm{~h}$, Fig. 1(b). We demonstrate that the mean wavelength (MW) and mean amplitude (MA) of the $\mathrm{Cr}$ concentration fluctuations increase from the unaged state during isothermal aging at $280{ }^{\circ} \mathrm{C}, 320{ }^{\circ} \mathrm{C}, 360{ }^{\circ} \mathrm{C}$, and $400{ }^{\circ} \mathrm{C}$ to $4300 \mathrm{~h}$. Both quantities increase a greater amount when aging at higher temperatures when compared to lower temperatures [5]. 


\section{References:}

[1] J.W. Cahn, Acta Metall. 9 (1961), p. 795.

[2] S.C. Schwarm et al, Mater. Sci. Eng. A. 680 (2017), p. 359.

[3] A. Devaraj et al, Inter. Mater. Rev. (2017).

[4] J. Zhou et al, Microanal. 19 (2013), p. 665.

[5] This work is supported by the Department of Energy - Nuclear Energy University Program (DOENEUP), contract number DE-NE0000724; Dr. Jeremy T. Busby, Oak Ridge National Laboratory (ORNL), technical monitor.
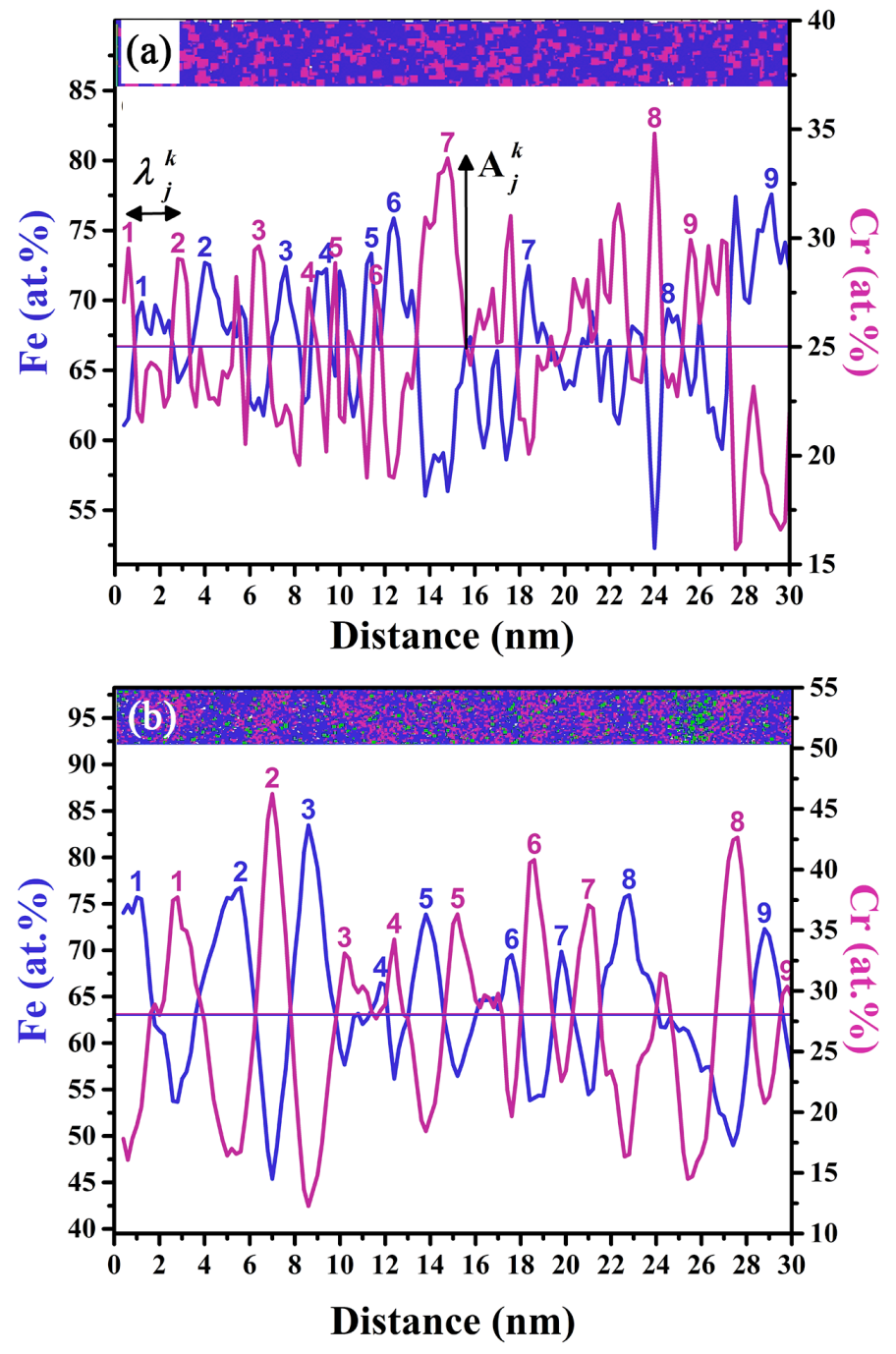

Figure 1. (a) An example region of interest (ROI) illustrating alternating Fe-rich $\alpha$-domains (blue) and $\mathrm{Cr}$-rich $\alpha$-domains (magenta) with corresponding $\mathrm{Fe}$ and $\mathrm{Cr}$ concentration profiles in unaged $\mathrm{CF}-8$ CDSS. (b) An example ROI illustrating alternating Fe-rich $\alpha$-domains (blue), Cr-rich $\alpha$-domains (magenta), and a Ni-rich G-phase precipitate (green) with corresponding $\mathrm{Fe}$ and $\mathrm{Cr}$ concentration profiles for $\mathrm{CF}-8$ specimen aged to $4300 \mathrm{~h}$ at $400{ }^{\circ} \mathrm{C}$. 